\title{
Temperature dependent volume expansion of microgel in nonequilibria
}

\section{A dynamic effect resembling the globule-coil percolation type crossover with a certain powerly conformable time-temperature decisive increment}

\author{
Adam Gadomski, Natalia Kruszewska ${ }^{a}$, and Piotr Bełdowski \\ Group of Modeling of Physicochemical Processes, Institute of Mathematics and Physics, UTP University of Science \\ and Technology, Kaliskiego 7, 85796 Bydgoszcz, Poland
}

Received 22 June 2018 / Received in final form 6 July 2018

Published online 8 October 2018

(C) The Author(s) 2018. This article is published with open access at Springerlink.com

\begin{abstract}
We propose to adopt a non-isothermal and colloid type cluster-cluster aggregation stochastic model aimed at comprehending the temperature driven and polymer swelling accompanying volume expansion encountered in microgels. The nonequilibrium nature of the process is captured by describing expansion characteristics with simplified power laws, indicating the scalability of properties with time and temperature. Additionally, molecular dynamics simulations of the presented mechanism for a chosen biopolymer have been performed. This can be of interest for experimenters working in the field of nonequilibrium phase transitions, and fairly prospectively, within the area of thermal phonon-involving technology. In these areas, scanning the system's temperature, or sometimes tuning similar dissipation-addressing physical factors, such as $\mathrm{pH}$, appears to be a fairly pivotal examination case.
\end{abstract}

\section{Introduction}

Microgels are defined as viscoelastic systems classified as intermediates between polymer chains, viz coils, and the so-called macrogels, such as gelatine or yoghurt [1,2]. They are often chemically prepared as two-component systems which consist of mixed solute and solvent phases. The solvent molecules interact with solute particles composed of polymer chains, and their aggregates, incline to behave similar to network-like structures [1]. Their complicated interaction map causes difficulty in achieving a thermodynamic equilibrium. Viscoelastic properties of such a system undergo some structural-geometric changes as a function of temperature and time. An example of such a temperature-dependent transformation is a microgel's volume expansion. The temperature conditions are able to influence, in the course of time, the suitability of a solvent for the solute molecules [3]. Good solvent conditions cause the polymer chains to expand in space due to solvent molecules' absorption thus creating coils. At a low temperature an opposite physicochemical scenario prevails. The solvent molecules interact poorly with the polymer chains, allowing for greater self-interaction which can result in globule formation. Thus, the temperature, playing a role of a control parameter, establishes a passage between coil and globule by decisively changing the solvent conditions.

\footnotetext{
${ }^{\mathrm{a}}$ e-mail: nkruszewska@utp.edu.pl
}

Such a physicochemical scheme is well-described by the Flory-Stockmayer theory. It is also well envisaged by the pivotal role played by the Flory-Huggins solute-solvent interaction energy parameter $[4,5]$.

From statistical-mechanical point of view the microgels are nonergodic, meaning that the corresponding time and ensemble averages performed on them take on different characteristics. It is also worth to mention that by adding certain ingredients to those two-component microgel systems one may change their basic interaction map, resulting in a modified aggregation propensity, as is the case with the introduction of specific monovalent or divalent cations $\left(\mathrm{Na}^{+} ; \mathrm{Ca}^{2+}\right)$ or macroions, e.g. proteins (gelatin), to the system [1].

At the nanoscale level, a crucial task arises when controlling heat dissipation within the competing globule and coil structural formations. This results from the slightly interconnected polymer network vibrations, known as thermal phonons in the field of solid-state physics [6]. Organizing the microgel as a whole in varying thermalbath conditions, mainly the tuning of the temperature step within the time span, emerges as the task of obtaining functional gelling materials of affordable (nonlinear) viscoelastic properties [6].

The article is organized in a following way. In Section 2, we try to unravel a model sol-like (typically, nonisothermal) system by employing a cluster-cluster analogy of colloid type (close-packed system) [7,8]. We assume here 
low temperature circumstances (system trapped in a low energy well). Such a system is supposed to conserve its total volume (or, for $d=2$ dimensional space, area [3]), and may remain nearly inactive as far as its overall spatial expansion is concerned (in the presented model we connect this global behavior to a polymer in its globular state which is a fairly local effect). In Section 3, after escaping from the low-value energetic depot by means of a controllable temperature raise, we are able to accomplish a spatial expansion of the system, which we wish to assign to a polymer in its coiled (loosely-packed) state [4]. Section 4 presents a molecular dynamics computer simulations used to show an importance of temperature in biopolymer's volume change. Section 5 provides a concise discussion of the micro-structural crossover addressed by the underlying study. It also presents an outlook of the approach applied, emphasizing the fact that the analogy addressed suits, in a qualitative manner, the nonergodic viscoelastic framework, particularly in the context of bioreactive gels and/or living-matter.

\section{Model of globular clusters at a low temperature}

The theoretical model, which is predisposed to reveal the thermal-activated microgel volume's expansion, assumes that the role of clusters is played by polymer globules occluded by the solvent molecules, constituting solventinvolving domains [8]. The model is proposed for a semiconcentrated polymer solution in which the solute and solvent (viz water) coexist at a relatively low temperature $T$, so the solvent is unable to cause the polymer globules to become coils. The reason of this is that the solvent molecules are not able to penetrate the polymer's interior and cause a swelling of the chain. At the other side, such poorly swollen polymers are capable of diffusing under dynamic structural (fairly restricted) confinement, both in terms of their mass-center (random walk) motions and rotational movements. They can also interact with each other yielding dimers, oligomers, and some aggregates. They can form a more or less cellular microstructure with well-separated but poorly hydrated polymer domains. The microstructure would, to a first approximation, remind a sol phase since the domains are relatively immobile and less reactive, due to their weak reactive encounters.

At a given temperature $T$, a hydrodynamic radius, $R_{h}$, of the globular (sol-like) polymers' chains is $t$-dependent, due to being prone to self-expansion and proportional to the effective domain-occupation volume $v$. It can be written, according to a simple geometric proportionality relation, as $v(t) \sim R_{h}^{3}(t)$. This postpones the form factor, e.g. the hydrated polymer domain, provided that we confine ourselves to hydrogels as an often invoked and popular example of microgels [1].

Local pressure differences are a driving force of the mass transfer between neighboring domains. The transfer is sometimes accompanied by the corresponding structural rearrangements of diffusive nature inside each domain [7]. Such domains resemble tightly built clusters or even "soft" grains that might have well defined surface-tension factors. The system (stochastic) dynamics can be described by the mass current $J(v, t)$ along the "reaction coordinate" $v$. The current form can be adopted from a model of cluster-cluster aggregation and its isothermal evolution $[7,8]$, and written as

$$
J(v, t)=-\frac{\partial}{\partial v}(D(v) \phi(v, t)),
$$

where $\phi(v, t)$ is the probability density of finding a domain of volume $v$ at time $t$, and $D(v)$ is a $v$-dependent (or, state-dependent) diffusion function. The diffusion function indicates quantitatively a colloid type cluster formation [8], thus, it is provided by $D(v)=D_{0} v^{2 / 3}$, where $D_{0}$ is introduced to preserve dimensionality. Note that, because of the simple geometric proportionality between the domain's hydrodynamic radius and its volume, $v^{2 / 3} \propto R_{h}{ }^{2}, D$ is constructed to be proportional to the domain surface, $s_{D}$, i.e. $s_{D} \propto R_{h}{ }^{2}$.

The right-hand side of the equation (1), after differentiation over $v$, can be expressed by

$$
J(v, t)=-D^{\prime}(v) \phi(v, t)-D(v) \frac{\partial}{\partial v} \phi(v, t),
$$

where $D^{\prime}(v)=\frac{2}{3} D_{0} v^{-1 / 3}$. Using once again the geometric proportionality between domain hydrodynamic radius and volume, we can state that $2 v^{-1 / 3} \sim \frac{2}{R_{h}}$. The constant $\frac{D_{0}}{3}$ again maintains proper physical units. The quantity $\frac{2}{R_{h}}$ stands for twice the mean curvature of the sol-type shrunken polymer domain of globular propensity. In each domain, there is some pressure difference, $\Delta \pi_{D}$, between external and internal parts of the domain. It follows the Kelvin-Laplace law, namely

$$
\Delta \pi_{D}=\sigma \frac{2}{R_{h}}
$$

in which $\sigma \propto D_{0}$ is the surface tension of the domain circumference. Bear in mind that additionally $\sigma=\sigma(T)$. Because the stochastic variable $R_{h}$ is $t$-dependent, the pressure $\Delta \pi_{D}$ (see Eq. (3)) changes over time $t$ within the sol-like but semi-concentrated (i.e. well packed or relatively dense [7]) structure during its swelling. Moreover, and still within our approximate reasoning offered (see discussion below Eq. (2)), the $\Delta \pi_{D}$ is certainly involved in the drift part of current $J(v, t)$ through the KelvinLaplace law (see Eq. (3)) of micro-capillarity. This holds because the linear size of polymer-solvent domains of volume $v$ belong to the submicron scale being of the magnitude of $100 \mathrm{~nm}$ [1]. Thus, it can be written that

$$
J(v, t)=-\Delta \pi_{D} \phi(v, t)-D(v) \frac{\partial}{\partial v} \phi(v, t),
$$

when one assumed that $\sigma=\frac{D_{0}}{3}$ applies [9]. The continuity equation for such a system reads $\frac{\partial}{\partial t} \phi(v, t)+\frac{\partial}{\partial v} J(v, t)=0$. Thereby, equation (4) should be completed by the socalled normal boundary conditions of absorbing type $\phi(v=0, t)=\phi(v=\infty, t)=0$, meaning that no grains' 
magnitude prevails during the system's evolution [8]. The simplest selection of the initial condition can be a delta Dirac distribution $[7,9]$.

Three important measures can be read out from such an approach of the pressure-drifted diffusion dynamics of the well packed sol-like and weakly reactive system: $n(t)$, the average number of the domains; $V(t)$, designating the total system volume; and $R_{D}(t)$, the average radius of the dehydrated globular domain.

The first two $(n(t)$ and $V(t))$ can be evaluated from the first and the second statistical moment given by an equation

$$
m_{i}(t)=\int_{0}^{\infty} v^{i} \phi(v, t) d v
$$

where $i=0,1,2, \ldots$ Thus, $n(t)=m_{0}(t)$ and $V(t)=m_{1}(t)$. The solution of $\phi(v, t)$ can be obtained using a variableseparation method, which has already been employed elsewhere [8]. The third quantity, $R_{D}(t)$, can be estimated based on a geometrical relation

$$
V(t) \simeq n(t) R_{D}^{3}(t)
$$

Hereby, $R_{D}{ }^{3}(t)$ corresponds to the average volume of a single domain. The averaging is performed as an integration over $v$, where $v \in[0, \infty]$, cf. equation (5).

The three key dynamic measures obey scaling laws at their asymptotic regimes for which $t \gg t_{0}$ (where $t_{0}$ is an initial moment). First, $n(t)$ conforms to a scaling law in the form of

$$
n(t) \sim t^{-\frac{3}{4}}
$$

Second, $V(t)$ is expected to be in accordance with a constancy condition [10], thus it scales trivially with $t^{0}$, i.e.

$$
V(t)=V\left(t_{0}\right) \rightarrow \text { const. }
$$

Third, $R_{D}(t)$ scales as

$$
R_{D}(t) \sim t^{\frac{1}{4}}
$$

Note that all equations above are proper in three dimensional (Euclidean) space. In a $d$-dimensional space the scaling goes as $R_{D}(t) \sim t^{1 /(d+1)}$. Let us emphasize that equation (6) is consistent with the scaling laws provided, cf. $[7,8]$. According to equation (8), the total volume $V$ is a conserved quantity upon such low-energy thermal conditions or because of being trapped in a low-energy well. Thus, the sol system is foreseen to be conservative and non-expanding, resembling in this way some stagnant and weakly reactive cellular network. In the network, however, nodes (globular domains) are nearly disjoint objects due to some non-negligible $\Delta \pi_{D}$-s distributed uniformly over the all system under study the values of which become constant.

Let us now come back to the pressure difference, $\Delta \pi_{D}$, and look on its distribution over the total volume $V$. It was shown (see Eq. (3)) that based on Kelvin-Laplace law, $\Delta \pi$ is proportional to $2 / R_{h}$. Using once again a geometric proportionality between the $R_{h}$ and $v$, the pressure difference can be provide as $\Delta \pi_{D}(v)=2 \sigma v^{-1 / 3}$. The equation, after common mathematical transformation, can be presented as $v=\left(2 \sigma / \Delta \pi_{D}(v)\right)^{3}$. Based on equations (5) and (8), an ensemble-averaged specific quantity can be evaluated $\left\langle 2 \sigma / \Delta \pi_{D}(v)\right\rangle^{3}=m_{1}(t)=V(t)=V\left(t_{0}\right)$. The average, due to the statistical uniformity of the system, reads $\left\langle 2 \sigma / \Delta \pi_{D}(v)\right\rangle^{3} \equiv(2 \sigma)^{3}\left\langle\Delta \pi_{D}(v)\right\rangle^{-3}=V\left(t_{0}\right)=$ const. We recall that the domain's surface tension $\sigma$ is assumed to be independent of $t$ during the evolution. It is in accordance with the fact that the domain borders are characterized by mainly $T$-dependent surface tensions. Thus, one is able to refer in full to the constancy of $\left\langle\Delta \pi_{D}(v)\right\rangle$ to yield the following

$$
\left\langle\Delta \pi_{D}(v)\right\rangle=\frac{2 \sigma}{\left[V\left(t_{\mathrm{o}}\right)\right]^{1 / 3}}=\text { const. }
$$

An internal mechanical stress, assigned to the polymeric system at the late-stage limit [11], may distribute uniformly in very similar way to the $\Delta \pi_{D^{-}}$s. For example, for an ideal (equilibrium) cellular network in a 2D space, envisaged by a honeycomb microstructure, the mechanical stress would distribute over the triple junction crossing points, nearly at the angle of $2 \pi / 3$. Nevertheless, in certain bubbles-containing (or, soap foam-like) analogous systems, the circumstance could be different [12].

\section{Nonconservative model system beyond the low thermal energy regime, promoting a formation of microgels}

Let us take for granted that some additional energy is put, in a controlled way, in the system by increasing $T$ gradually, say, from some $T$ to a $T+\langle\Delta T\rangle$, wherein $\langle\Delta T\rangle>0$ is an averaged temperature step, to be discussed in next paragraphs. The solvent (viz. water) molecules, being more translationally mobile than the polymer chains, will explore their degrees of freedom and diffuse, performing random walks at rate determined by the temperature and structural conditions. The structural conditions, attributed to non-bulk states of water molecules, e.g. the bound and the interfacial ones, suggest a departure from classic Fickian (diffusional) behavior. Such behaviour is of high practical relevance to drug release in microgels [13]. The addition of $\Delta T$ thus instigates increased penetration of polymer chains, triggering transition of globule structures into coils. As coils dynamically occupy more space, this transition manifests an increased volume of the entire sol-like system. It is possible to show that $V(t)$ would scale nontrivially as [14],

$$
V(t) \sim t^{\frac{1}{2}}
$$

equation (11) demonstrates the nonconservative character of the formation, thus, in such semi-quantitative terms the thermal expansion of the volume upon increasing $T$, very 
characteristic of microgelling systems, has been established. According to reference [8] the gelling system is more entropy-productive than its low $T$ counterpart. It is physically legitimate, since in the current $J(v, t)$, after engaging the domain-wise thermal expansion, taken here as a fair analog to the crossover from globule to coil [1], one precludes the drift term $\Delta \pi_{D} \phi(v, t)$ from being non-negligible as compared to its diffusive counterpart $-D(v) \frac{\partial}{\partial v} \phi(v, t)$ in the overall current (4). It then results, due to $\Delta \pi_{D} \approx 0$ in the long time $\left(t \gg t_{o}\right)$ domain and on the local scale, in the pressure average $\left\langle\Delta \pi_{D}(v)\right\rangle \sim \frac{2 \sigma}{t^{1 / 6}}$, while keeping exclusively one diffusive term in the subsequent current, i.e.

$$
J(v, t)=-D(v) \frac{\partial}{\partial v} \phi(v, t)
$$

Note that, according to the Kelvin-Laplace law, equation (3), $\Delta \pi_{D} \approx 0$, appearing locally, means that first the surface tension of the domain $(\sigma)$ has to be lowered because of very relaxed more solution-than-solute conditions. In addition, the domains would ultimately flatten, owing to the very small value of the curvature $\kappa_{D}$, reflecting qualitatively the nature of the microgelling system, which diffusively expands [1]. In other words, the domains, viz. coils, are simply much more swollen, thus the surface tension within the coil/domain and at its shell approach the same value. Before, i.e. in conservative conditions, the situation was quite different - the surface tension of the globule's core was bigger than at the globule's shell.

Mutatis mutandis, the number of polymer-solvent microdomains present in the microgel [10] scales as

$$
n(t) \sim t^{-\frac{1}{4}}
$$

thus decreasing more gradually in time than for the volume-conservative system.

From the above it follows that the scaling for the average radius, $R_{D}$ is unchanged, equation (9). The exponent of $1 / 4$ involved in the scaling relation (9) keeps the signature of $(d+1)$ random close packing, a measure characteristic of a $d$-dimensional geometrical-physical space upon confinement. Recall that the $d$-dependent exponent is $\nu_{\mathrm{CP}}(d)=1 /(d+1)[10]$.

It can also be foreseen that for the thermally sensitive and volume expanding model microgels, i.e. in high $T$-conditions, one would expect a relationship between the average temperature step $\langle\Delta T\rangle$ and the effective time span of the corresponding volume expansion of the microgel. An argumentation for this relation follows. First, let us refer to what has been obtained for the average Laplace pressure in the system, $\left\langle\Delta \pi_{D}(v)\right\rangle \sim \frac{2 \sigma}{t^{1 / 6}}$, which is going to attain very small values when $t \gg t_{o}$ holds. It is due to the (free) expansion of the system as a whole, provided that no external load viz cause would perturb it. It is also consistent with the observation that the ideal or smart microgels ought to be highly swellable and well stretchable. For high $T$, which is the case, such a minimally constrained system has to obey the ideal-gas state equation. By this rationale, one would - as a first approximation - write down $\left\langle\Delta \pi_{D}(v)\right\rangle V(t)=n R\langle\Delta T\rangle$, wherein $n$ is the number of moles of the solution, causing the polymer system to swell, and $R$ is the gas constant. The product of $M(t)=$ $\left\langle\Delta \pi_{D}(v)\right\rangle V(t)$, according to equation (11) scales with $t$ as $M(t) \sim t^{1 / 3}$, as does $\langle\Delta T\rangle \equiv\langle\Delta T(t)\rangle$, namely

$$
\langle\Delta T(t)\rangle \sim t^{\frac{1}{3}}
$$

Realize that relation (14) interconnects time $(t)$ and temperature step $(\langle\Delta T(t)\rangle)$. Therefore, it may be thought of constituting an analog of time-temperature superposition (Williams-Landel-Ferry model) characteristic of glassy polymeric systems, when tuning their dynamic, thus viscoelastic behavior, toward the glassy temperature [11]. (There is one precaution to this interference: the surface tension $\sigma$, involved in (3), has to remain independent of $t$ at this scale of observation.) It seems natural, since one has to wait for $\langle\Delta T\rangle$ to be effective in order to cause a suitable volume expansion. Notice that relation (14) is nonlinear in time, presumably, pointing indirectly to the nonergodicity of the system. Based upon equation (14), one is able to think of some ontology of temperature in systems that suffer from interactiongeometry, here viscoelasticity containing gel-like obstacles, to attain a (local) thermodynamic-equilibrium state [15]. Memory effects have seen indicated to support such scenario [16]. It also seems possible to manoeuvre by means of an external pressure and control the involved system size (toward domain volume, a local-scale effect) [9]. After achieving small-size domains in the microgel, as is the case of smart hydrogels, one would get highly responsive elastic, thus functional, material [17]. On the other hand, one is urged to lose the notion of microcapillarity when the linear sizes of the domains, $R_{h}$ (see, beginning of Sect. 2) become too small, enforcing in practice a zeroth Laplace pressure effect in equation (3). The system then will land ultimately on the purely diffusive gas-like and domain-less limit, becoming unpredictable.

It is worth mentioning that the power-law behavior of the time-temperature relation (but with different exponent $T(t) \sim t^{0.15}$ ) has also been noticed in other (experimental) studies considering thermomechanical analysis of polymers, which measured deformation of a material contacted by a mechanical probe. Thermomechanical analysis penetration tests, used to detect relative states of cure of a thermoset polyester, show that the penetration temperature under a fixed load increases with cure and can be used as a measure of the degree of cure of an elastomer compound (cf. Fig. 30 in [18]). Thermogravimetry, another experimental method suitable for detecting the amorphous gel-like phase in terms of time-temperature measurements, is used convincingly for uncovered membrane structure examination in [18].

Moreover, power laws in oscillating frequency domains [19], as well as time-temperature relationships of qualitatively similar types, have also been presented by [20] for agar (bio)gels/hydrogels, cf. Figure 5 therein. For a review, specifically addressing stereocomplex gels with microcrystalline domains, cf. an SEM photograph on Figure 20 therein, one is encouraged to consult [21] in which case (poly)lactides uncover their versatile timetemperature entailed dynamics of microgel formation. 


\section{Numerical realization of the importance of temperature in biopolymer swelling}

In order to check the importance of temperature in biopolymer swelling, a molecular dynamics simulation has been performed, using YASARA Structure Software (Vienna, Austria) environment [22]. Hyaluronic acid (HA) has been chosen as a simulated object. Studies on this biopolymer are very rife due to the importance of its role in biological systems such as e.q. in articular cartilage (AC), in which lubrication is highly affected by quantity and quality of the HA chains within synovial fluid [23]. In $\mathrm{AC}$ the temperature can increase as much as $10-15 \mathrm{~K}$ as a result of external load applied to it [24]. Such a temperature's change causes alterations in physicochemical conditions of the system. If the change is directional, certain thermodynamic cross-effects can lead to Ludwig-Soret type co-effects [25], altering the corresponding piece of the microgel. These effects can orient the system toward a particular order, allowing certain microcrystallinity to emerge. Another interesting microstructural option may be offered upon invoking the so-called plum-pudding gel model, containing microgel inclusions, in which Fickian vs. non-Fickian effects compete readily [13].

The computer simulation of changes in HA conformation, in a course of time and temperature increase, has been constructed as follows. Sixteen formed-in-sol HA chains have been immersed in a water solution. Afterwards, molecular dynamics simulations were performed under three temperatures (chosen to be around physiological temperatures): 300,310 and $320 \mathrm{~K}$. All-atom molecular dynamics simulations were performed using AMBER03 force field to evaluate interactions between HA chains [26]. Time steps were set to $2.5 \mathrm{fs}$ and simulations allowed to run for $10 \mathrm{~ns}$ (what gives $4 \times 10^{6}$ simulation steps). All three simulations were performed at the same conditions: $\mathrm{pH}=7.0$ and $0.9 \% \mathrm{NaCl}$ aqueous solution. Initial structures have been formed as highly folded chains (sol form), while with time (10 ns of simulations) they showed up some level of uncoiling (see Fig. 2). Time evolutions of the radius of gyration, $R_{g}$, have been presented in Figure 1 (note, that the $R_{g}$ values have been rescaled by initial radius $R_{g 0}$, thus presented values show a relative factor of expansion). All results are averaged after 20 realizations. When temperature increases, the rate of $R_{g}$ 's change also increases, establishing temperature as an important factor determining shape and crosslinking properties of HA network. Fitting functions show that $R_{g} / R_{g 0}$ increases in time according to power law $R_{g} \propto t^{\alpha}$, where for temperatures: $300 \mathrm{~K} \alpha=0.802 \pm 0.010$ $\left(R^{2}=0.9972\right), 310 \mathrm{~K} \alpha=0.602 \pm 0.018\left(R^{2}=0.988\right)$ and $320 \mathrm{~K} \alpha=0.541 \pm 0.015\left(R^{2}=0.991\right)$. Thus, it can be clearly seen that in temperature $320 \mathrm{~K}$ the HA globules swell much faster than in $310 \mathrm{~K}$.

\section{Summary}

In this study, a kinetic-thermodynamic depiction of a model sol-gel phase change has been unveiled in terms of a statistical-thermodynamical concept $[7-9,14]$. To achieve the goal, a cluster-cluster aggregation framework,

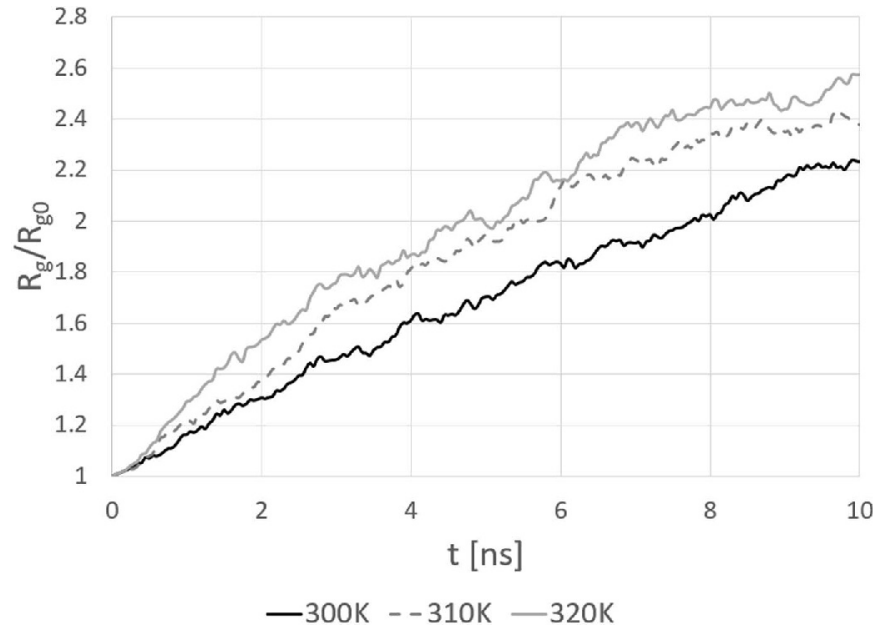

Fig. 1. Radius of gyration as a function of time of a single HA chain in three temperature values.

describing a drifted and diffusive nature of the system, has been taken to semi-quantitatively model the temperature dependent, and polymer swelling assisted, expansion of the gelling (viz. percolation-like) phase. The modeling has been performed with the aim of revealing some basic trends of the hybrid microgel formation, uncovered in [1]. Nevertheless, we are aware that there exist reverse processes which are not captured by presented model $[27,28]$.

A certain novelty emerges from the moments in our statistical approach (see Eq. (5)). They appear to lead to a precise estimation of the average Laplace's pressure, $\left\langle\Delta \pi_{D}\right\rangle$. This turns out to be a constant value (Eq. (10)) for the volume-conservative sol-like phase, and in turn, decreases slowly as function of time $(t)$, namely $\left\langle\Delta \pi_{D}(v)\right\rangle \sim 2 \sigma / t^{1 / 6}$. In reference [1], one may find some indications about microgelling systems that tend to equilibrate over a time span of about a few days.

It is useful to estimate the internal mechanical stress' values, indicative of the microgel and denoted by $\left\langle\sigma_{M}\right\rangle$ (an ensemble average value too), in terms of the homologous properties in relation to the Laplace's pressure, $\Delta \pi_{D}$. To a first approximation, it is possible to do under the conjecture $\left\langle\sigma_{M}\right\rangle \propto \Delta \pi_{D}$. If such a general assertion is accepted, one obtains

$$
\left\langle\sigma_{M}(t)\right\rangle \sim t^{-\frac{1}{6}},
$$

which produces a slow descent with time $t$. In a previous study on colloid-type aggregation [8], it has been hypothesized that for evaluating $\left\langle\sigma_{M}\right\rangle-\mathrm{s}$, based upon analogy with metallic or ceramic polycrystals $[9,14]$, that one could take $\left\langle\sigma_{M}(t)\right\rangle \propto 1 /\left[R_{D}(t)\right]^{\mu}$, wherein $\mu \simeq 1 / 2$ (the so-called Hall-Petch-Griffith characteristic exponent $[9,14]$, responsible for toughness and/or brittleness of the material) for $t \gg t_{o}$ when a final microstructure emerges. In our case, due to the scaling (9), we have to choose $\mu=2 / 3$, in agreement witch the surface-tovolume exponent, involved in $D(v)$ in the relation (1). The exponent meets the characteristics of colloid type systems. The overall mechanical-stress exponent is given 


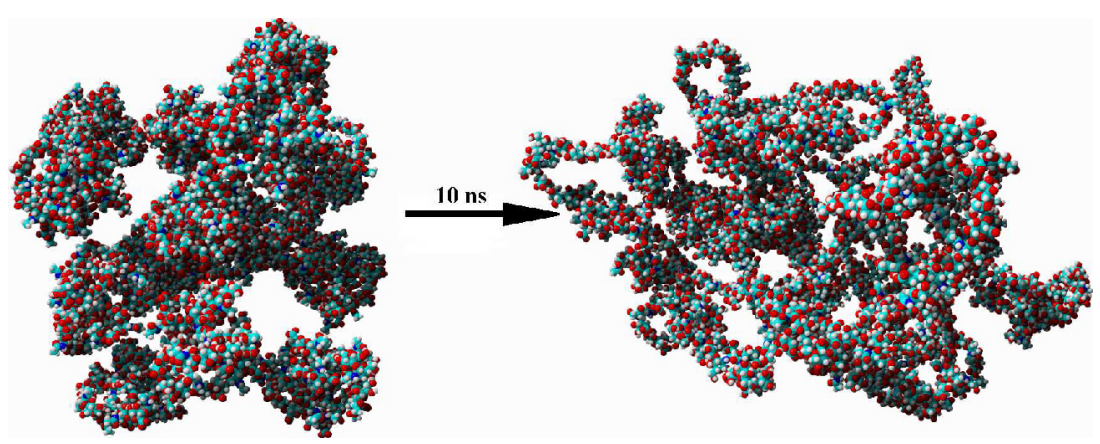

Fig. 2. Initial (sol) and final (gel) structure of HA network - result of molecular dynamics computer simulation. At the beginning HA chains were highly folded into globular form. Then, after some time in temperature $310 \mathrm{~K}$, the system starts uncoiling, thus creating gel-like structure. HA is composed of carbon colored cyan, hydrogen - white, oxygen - red, nitrogen - dark blue.

by $\mu_{M}(d)=\frac{d-1}{d} \frac{1}{d+1}$, bearing dimensional signature of the microgel's close packing $(d+1)$. Therefore, for thermally expanding and swollen microgels, two physical facts must be equally important: their colloidal surface-to-volume characteristics as well as the corresponding close packing conditions [1]. The equation above seems to reflect this natural association.

The value of $\mu=2 / 3$, introduced in $D(v)$ in equation (1), would also suggest an interpretation of the field $\left\langle\sigma_{M}(t)\right\rangle$ propagation through inter-domain spaces ("grain boundaries") in terms of phonons wandering randomly along them. This so-called random-walk exponent should be here taken as $\mu_{R W}=1 / \mu$. The mean squared displacement of the phonon bursts, $\left\langle r_{M S}{ }^{2}(t)\right\rangle$, generated when the mechanical stress propagates thorough the interspaces could then be estimated as

$$
\left\langle r_{M S}^{2}(t)\right\rangle \sim t^{2 / \mu_{R W}}
$$

thus, resulting in a scaling behavior of $\sim t^{4 / 3}$, meaning that the phonons penetrate in an accelerated diffusive way to the underlying interspaces [6]. This suggests the notion of self-avoiding random walks of the (thermal) phonons, under the assumption that the interspaces are just quasi $2 \mathrm{D}$ surfaces. In this vein, the exponent $\mu_{R W}$ is a characteristic exponent of the self-avoiding random walk (abbrev. by SARW), suggestive of minimally collisional motions of the phonons in the planar interspaces. Of course, the exponent $\mu_{\mathrm{SARW}}=4 / 3$ according to Flory-Fisher mean-field theory [29].

For their practical usefulness in nonergodic systems ${ }^{1}$, the scaling law and the interconnections between their exponents are presented in Table 1. Their interdependence is apparent when looking at how the statistical moments scale (Eq. (5)), as well as how a

\footnotetext{
${ }^{1}$ Realize that to a certain extent the nonergodicity is quite tacitly drawn in terms of time-temperature or $t$ vs. $T$ superposition (or, equivalence) principle, characteristic of amorphous glassy and gelling systems [29]; therefore, one anticipates some difference in the "cumulative" Laplace's pressure for both systems of interest, revealing that the nonconservative system suffers from appreciable difficulties to equilibrate in a reasonable time span (cf. Eq. (15)). A forefront's manifestation of nonergodicity in microgelling systems can be conjectured when based on the nonlinear relation (14).
}

Table 1. Model-estimated values for the different exponents, characterizing the (non)conservative system capable of mimicking a crossover (or, a certain immature phase change) from the globule to coil state, and reversely [1], characteristic of the volume-expansive (in particular, weekly interconnected [16]) microgels.

\begin{tabular}{ccccc} 
Nonconser & $R_{D}$ & $n$ & $V$ & $\sigma_{M}$ \\
-vative: & $\nu_{R_{D}}=\frac{1}{4}$ & $\nu_{n}=\frac{1}{4}$ & $\nu_{V}=\frac{1}{2}$ & $\nu_{\sigma_{M}}=\frac{1}{6}$ \\
microgels & Eq. (9) & Eq. (13) & Eq. (11) & Eq. (15) \\
& & & & \\
\hline Conservative: & $R_{D}$ & $n$ & $V$ & $\sigma_{M}$ \\
sol-type & $\nu_{R_{D}}=\frac{1}{4}$ & $\nu_{n}=\frac{3}{4}$ & $\nu_{V}=0$ & $\nu_{\sigma_{M}}=0$ \\
phase & Eq. (9) & Eq. (7) & Eq. (8) & Eq. $(10)$
\end{tabular}

simple geometrical proportionality relation applies, cf. equation (6).

Another study demonstrates to a great degree a firm working analogy between gelling/percolating [29] and prone-to-aggregation viscoelastic assemblies $[2,16]$, and justifies some solid efficiency of the random-walk approximation employed.

Our work tries to build scalable and controllable methods for manipulating the thermal-bath, or temperaturescanning under time-temperature sensitive conditions. It is relevant toward obtaining micro-gelling functional materials when the mechanism of mechanical properties originates in the nanoscale microstructural arrangement of the system $[6,11]$.

The scaling law, expressed by equation (14), and all interrelated asymptotic time-involving and thermodynamically accessible algebraic relations, such as those of micro-elastic and non-conservative propensities, cf. equation (15), can establish a processing avenue for food gels, such as yoghurts, evolving gradually with in-time raised temperature (virtually, structure-spoiling) step. Based on equations (14) and (15), one may entirely control the process of within-gel slow stress relaxation (Eq. (15)) upon controlling the temperature increase, cf. equation (14) such that the product of them, would 
asymptotically approach a (reasonably) minimal value. This would imply that such a controllable and stepwise temperature raise can by no means make the internal mechanical stress field behave uncontrollably, since the minimal-value argument is assumed to apply further on. This same argumentation can presumably be addressed qualitatively to thermoresponsive biopolymers, and in principle, to living cellular tissue [30]. Put another way, if the above addressed minimal-value criterion is not distinctly overridden, there seemingly appears no destruction, nor even a denaturation of the proteinous smart (functional), specifically yoghurt-type biomaterial phase, under certain gradual thermal agitation $[1,17]$.

Let us notice that dynamic and network-involving scenarios of microgel types appear inevitably at least from two areas of the approach employed. First, in the biophysical area of ultralow friction and facilitated lubrication of articular cartilage(s), see [31], wherein the network-like constructs of HA respond synergistically to the external load's action. Second, when within a cell the (anomalously bioreactive) metabolic pathway spreads out over its complex viscoelastic interior in intimately networking, and fairly dynamically organized manners, see [32].

To preview how the theoretical considerations correspond to a model microgel systems, molecular dynamics simulations have been performed. Presented results show that a rate of swelling of the hyaluronic-acid globules significantly increase together with temperature. Radius of gyration, in all simulated temperatures, increases in time according to the power law $R_{g} \propto t^{\alpha}$ with $\alpha<1$.

The structure-property paradigm in organic-inorganic nanomaterials and dimethacrylate POSS type networks has been disclosed via anomalous viscoelastic properties of time-temperature characteristics in [33] (see Scheme 3 and Figs. 7-9 and Eq. (1) therein).

A support of the present study by BS 39/2014 (UTP Bydgoszcz) is to be emphasized. AG benefited much from preliminary discussions with Prof. T. Wysocki (Nebraska Lincoln). Authors also want to thank S. Yuvan (ECU, Greenville) for his impact in obtaining the final form of the paper.

\section{Author contribution statement}

All authors were involved in the design and development of the presented research. All authors read, approved and revised the final manuscript.

Open Access This is an open access article distributed under the terms of the Creative Commons Attribution License (http://creativecommons.org/licenses/by/4.0), which permits unrestricted use, distribution, and reproduction in any medium, provided the original work is properly cited.

\section{References}

1. H. Cheng, G. Zhang, Thermally sensitive microgels: from basic science to applications, in: Hydrogel Micro and
Nanoparticles, edited by L.A. Lyon, M.J. Serpe (WileyVCH Verlag GmbH and Co. KGaA, Weinheim 2012)

2. D. Vlassopoulos, E. Stiakakis, M. Kapnistos, Rheol. Rev. 2007, 179 (2007)

3. N. Kruszewska, A. Gadomski, Physica A 389, 3053 (2010)

4. P.J. Flory, Principles of Polymer Chemistry (Cornell University Press, Ithaca, NY, 1953)

5. W.H. Stockmayer, Makromol. Chem. 35, 54 (1960)

6. N. Li, J. Ren, L. Wang, G. Zhang, P. Hänggi, B. Li, Rev. Mod. Phys. 84, 1045 (2012)

7. A. Gadomski, Philos. Mag. Lett. 70, 335 (1994)

8. A. Gadomski, J. M. Rubí, J. Luczka, M. Ausloos, Chem. Phys. 310, 153 (2005)

9. I. Santamaría-Holek, J. M. Rubí, A. Gadomski, J. Phys. Chem. B 111, 2293 (2007)

10. A. Gadomski, EPL 89, 40002 (2010), and references therein

11. D.W. Van Krevelen, in Properties of Polymers. Correlations with Chemical Structures (Elsevier, Amsterdam, 1972), Chap. 9

12. H. Lhuissier, D. Lohse, X. Zhang, Soft Matter 10, 942 (2014)

13. I. Lynch, K.A. Dawson, J. Phys. Chem. B 108, 10893 (2004)

14. M. Niemiec, A. Gadomski, J. Łuczka, L. SchimanskyGeier, Phys. A 248, 365 (1998)

15. A.V. Popov, R. Hernandez, J. Chem. Phys. 126, 244506 (2007)

16. E. Sarmiento-Gomes, I. Santamaría-Holek, R. Castillo, J. Phys. Chem. B 118, 1146 (2014)

17. L.-W. Xia, R. Xie, X.-J. Ju, W. Wang, Q. Chen, L.-Y. Chu, Nat. Commun. 4, 2226 (2013)

18. R.P. Chartoff, A.K. Sircar, Thermal analysis of polymers, in: Characterization and Analysis of Polymers (John Wiley and Sons, New Jersey, 2008)

19. S. Burov, M. Gitterman, Phys. Rev. E 94, 052144 (2016)

20. S. Boral, A. Saxena, H.B. Bohidar, J. Phys. Chem. B 112, 3625 (2008)

21. H. Tsuji, Macromol. Biosci. 5, 569 (2005)

22. E. Krieger, G. Vriend, J. Comput. Chem. 36, 996 (2015)

23. M.E. Blewis, G.E. Nugent-Derfus, T.A. Schmidt, B.L. Schumacher, R.L. Sah, Eur. Cell Mater. 13, 26 (2007)

24. M.N. Moghadam, P. Abdel-Sayed, V.M. Camine, D.P. Pioletti, J. Biomech. 48, 370 (2015)

25. A. Gadomski, N. Kruszewska, Phys. Lett. A 378, 2881 (2014)

26. Y. Duan, C. Wu, S. Chowdhury, M.C. Lee, G. Xiong, W. Zhang, R. Yang, P. Cieplak, R. Luo, T. Lee et al., J. Comput. Chem. 24, 1999 (2003)

27. T. Śliwa, M. Jarzȩbski, Curr. Top. Biophys. 37, 29 (2015)

28. L. Wang, X. Shi, J. Wang, Soft Matter 14, 3090 (2018)

29. M. Doi, S. F. Edwards, The Theory of Polymer Dynamics (Clarendon Press, Oxford, 1988)

30. S. Lindman, I. Lynch, E. Thulin, H. Nilsson, K.A. Dawson, S. Linse, Nano Lett. 7 , 914 (2007)

31. Z. Pawlak, W. Urbaniak, A. Oloyede, Wear 27, 1745 (2011)

32. A.J. Jezewski, J.J. Larson, B. Wysocki, P.H. Davis, T. Wysocki, Biotechnol. Bioeng. 111, 2454 (2014)

33. S. Bizet, J. Galy, J.-F. Gérard, Macromolecules 39, 2574 (2006) 